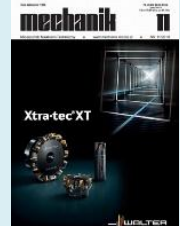

\title{
Novel geometric methods in multiscale analysis: curvature and slope
}

\author{
Nowe metody geometryczne w wieloskalowej analizie \\ topografii powierzchni: nachylenie i krzywizna
}

TOMASZ BARTKOWIAK *

The aim of this paper is to demonstrate the use of novel geometric multiscale methods in the characterization of surface topographies with a view to establishing functional relations between formation processes and resulted topography or between topography and its performance during interaction with the environment (e.g. friction, fatigue or gloss reflectance). Geometric properties of rough surfaces change with the scale of observation, i.e., measurement or calculation. In this paper, the author focuses on two novel method, which analyse slope and curvature in multiple scales. The digital nature of measured surfaces is recognized and is not smoothed to fit a continuous function to the measured heights. Curvature is represented as a second order tensor, which components describe maximal and minimal curvatures and their directions. In order to describe curvature distributions for analyzed regions, statistical parameters, such as: mean and standard deviation, are used. Those measures are utilized to find correlations between them and certain formation or performance parameters. The other method described here bases on slope. In this work, two calculation techniques are presented using orientation normal vectors estimated through covariance matrix method or cross product. As a result of further processing, 3D distributions of direction cosines are obtained, based on which multivariate statistical parameters are calculated (incl. measures of dispersion and higher statistical moments). This paper also presents the sample strong correlations between slope or curvature, for actual measured textures, and manufacturing or performance parameters. This indicates a great potential of those two methods in the development of multiscale characterization of surface topographies.

KEYWORDS: surface metrology, multiscale methods, curvature, slope, correlations

Surface topography affects a number of phenomena related to the interaction of this surface with the environment. At the same time, most of the manufacturing

* Dr inż. Tomasz Bartkowiak (tomasz.bartkowiak@put.poznan.pl)

- Instytut Technologii Mechanicznej, Politechnika Poznańska processes influences the topography of the surface. Topography consists of individual physical features of various sizes (such as blisters, grooves or microcraters) and often appears different when it is observed on a different scale of measurement. There is therefore a need for multiscale considerations.

The concept of scale can have multiple meanings. In metrology, it most often refers to the ratio of the distance on the object resulting from the measurement of the actual surface to the correct distance on the actual surface.

In the new meaning, the scale is a window through which the object is observed [1]. A measurement is a set of a specific range of wavelengths or frequencies. The notion of scale is often identified with size or dimension which results from the fact that certain surface features (scratches, pits, pores, etc.) of a given size or dimension can be best perceived when observed on a scale or range of scales. The scale may result from the measuring instrument used and in this case depends on the surface sampling interval. It can also be calculated depending on the multi-scale method used.

Physical phenomena dependent on surface topography can be associated with interactions that occur at several scales of interaction. Changing the surface topography as a result of the manufacturing process or wear can also occur at several scales. Therefore, it is crucial to understand the relationships between surface topography and processes during its production or its interaction with the environment.

These relationships will depend on the method of surface measurement, analysis of the measurement result and its characterization on many scales. Characterization means a numerical description of the measurement data.

The surface topography may vary depending on the scale of its observation. Significant features of surface topography, affecting its behavior during interaction with the environment, may refer to its form, waviness, roughness or microroughness. The irregularity of the surface topography causes that the values of the parameters describing the topography in a given scale depend on the scale of the measurement or on the computational scale. Most often only the scale group is important (in other words: its narrow range), for which a significant influence of a given parameter describing the topography on its interaction with the environment or the parameter of the manufacturing process is noted on the parameter describing the obtained topography. 
TABLE. Characteristics of currently used multiscale methods [1]

\begin{tabular}{|c|c|c|c|}
\hline Type & Geometric dimension & Large-scale parameter & Scale derivative \\
\hline \multicolumn{4}{|c|}{ Related to the dimension } \\
\hline 1D & Length & Relative length & Length-scale type complexity \\
\hline 2D & Surface area & Relative area & Area-scale type complexity \\
\hline $3 \mathrm{D}$ & Volume (filling) & Surface texture depth & Unused so far \\
\hline \multicolumn{4}{|c|}{ Derivatives } \\
\hline 1. order & Slope & Unused so far & Unused so far \\
\hline 2. order & Curvature 2D & One-dimension statistics for the profile & Unused so far \\
\hline 2. order & Curvature 3D & Statistics for tensor parameters & Unused so far \\
\hline
\end{tabular}

The currently used multiscale methods include:

- conventional methods in which measurements are made in many scales; measuring in one scale and using the Fourier transform; measurement in one scale and filtration using a morphological filter [2];

- the measurement method in one scale and the use of wavelet transform [3, 4];

- measurement method in one scale and the use of a sliding bandpass filter [5];

- measurement method in one scale and the use of a structure function [6];

- geometric methods [7, 8].

In the case of geometric methods, it can be noticed that the geometrical properties of rough surfaces change with the scale of observation or computational scale. A list of geometric methods is included in the table [1]. Derivatives are treated as finite differences, because measurement data is usually a set of points, not a continuous signal.

\section{Curvature}

The curvature of the surface is currently an important issue in the multiscale analysis. Like the heights, the curvature of the surface changes with the scale, but has the unique feature that it does not change its value depending on the reference system (datum). For the first time in a multi-scale analysis, the curvature of the surface was used for a multiscale description of roughness profiles of surfaces treated by shot blasting. The profiles were exported from three-dimensional surface measurements made with a confocal microscope. The curvature for the profile was calculated using the Heron formula for three neighboring points or using a differential notation for the curvature of the 2D curve. The curvature can be characterized by concave or convex areas, as indicated by the sign of curvatures of the main areas.

In the case of surface measurements, it is possible to determine the curvature tensor $\mathbf{T}$ for a given region of the measured texture. The curvature tensor can be represented as a $3 \times 3$ matrix:

$$
\mathbf{T}=\mathbf{P} \cdot \mathbf{D} \cdot \mathbf{P}^{-1}
$$

where:

$$
\begin{aligned}
\mathbf{P} & =\left(\mathbf{k}_{1}, \mathbf{k}_{2}, \mathbf{n}\right) \\
\mathbf{D} & =\left[\begin{array}{ccc}
\kappa_{1} & 0 & 0 \\
0 & \kappa_{2} & 0 \\
0 & 0 & 0
\end{array}\right]
\end{aligned}
$$

The sizes $\kappa_{1}$ and $\kappa_{2}$ are the main curves (respectively maximum and minimum). The eigenvectors $\mathbf{k}_{1}$ and $\mathbf{k}_{2}$ are the principal directions around which the surface bends the most and least. The vector $\mathbf{n}$ is a normal vector to the two principal directions mentioned $\mathbf{k}_{1}$ and $\mathbf{k}_{2}$ (fig. 1).

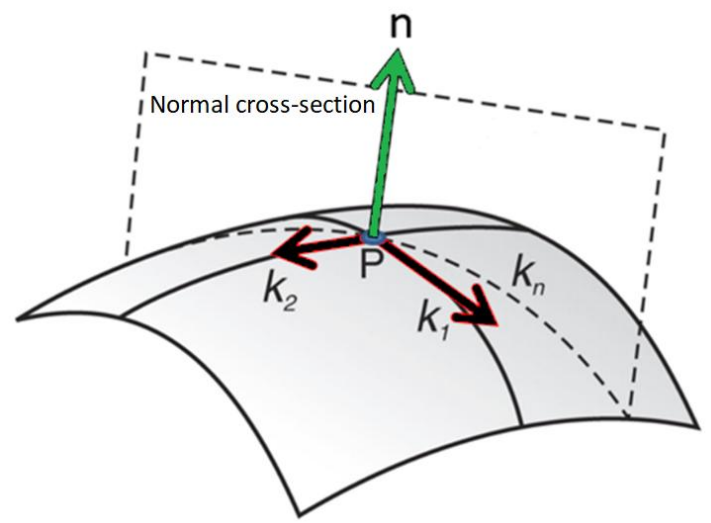

Fig. 1. Main directions of the curvature tensor and the vector normal to them

The combination of maximum and minimum curvature is also used, i.e. the mean $(H)$ and Gaussian $(K)$ curvature, which are given by the formulas:

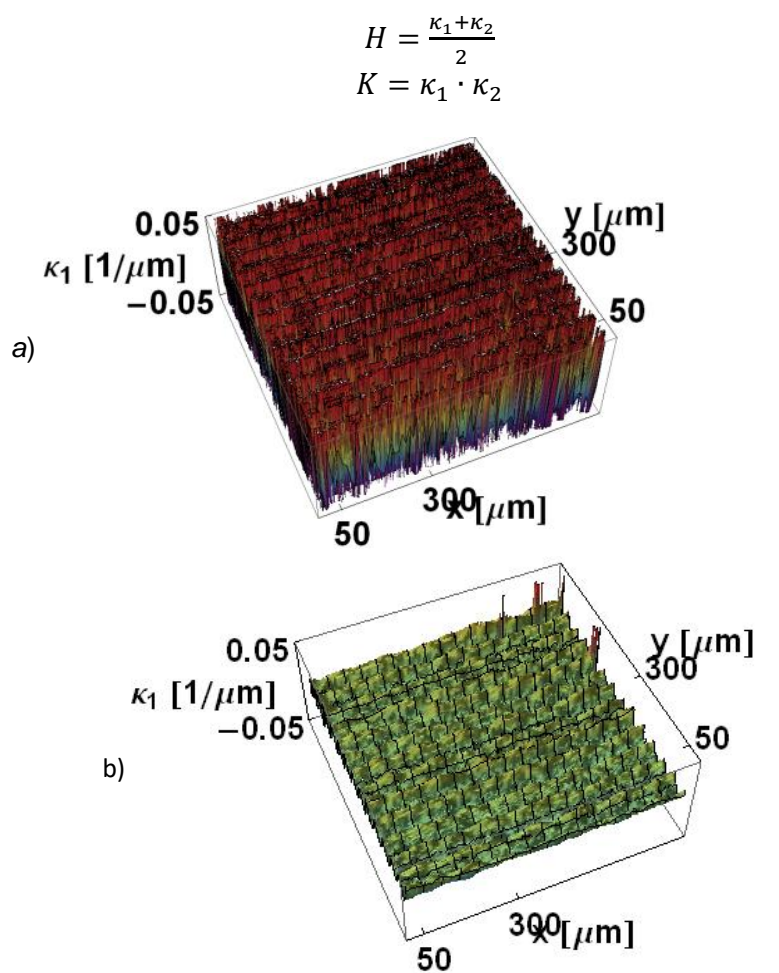

Fig. 2. Maximum curvature $\kappa_{1}$ of the area after the directional treatment, calculated for two scales: a) $1.92 \mu \mathrm{m}, b) 9.61 \mu \mathrm{m}$

The work [9] presents a set of parameters describing the distribution of maximum, minimum, average and Gauss curvature. These parameters are analogous to the parameters describing the distribution of the height of the measured points of the topography, such as $R a, R q$ or $S a$ and $S q$. 
Examples of curvature distributions for measured samples after conventional machining for two different scales are shown in fig. 2.

The multiscale analysis was successfully used to describe the surface topography obtained by the additive method [10] and after the microEDM treatment - in this case, strong functional correlations between the discharge energy and the resulting surface curvature were found [11]. Currently, work is in progress on the subsequent applications of this parameter to describe the directivity (anisotropy) of the surface and better description of other surfaces treated with unconventional methods.

\section{Slope}

Similarly as in the case of curvature, along with the scale and position $(x, y)$, the surface slope also changes. Until now, this quantity has not been used, apart from geology and oceanography $[12,13]$, in the multiscale analysis. According to ISO 25178, the RelA parameter (relative area) is based on the inclinations of the consecutive points, expressed by the cosecant of the inclination angle. Analysis of the slope distribution for the entire measurement data set, similarly to the analysis of the distribution of the measured heights, makes it possible to determine the statistical parameters of these distributions, and then to correlate them with the parameters of the manufacturing process or interaction.

The author proposes that in the case of surface measurements for each computational or measurement scale, determine for the measured point cloud the normal vectors for these points or for triangular elements resulting from triangularization of the point clouds (fig. 3 ).

There are many methods for calculating normal vectors for a point cloud. In this article, for the purpose of comparison, multi-scale methods are presented, where normal vectors are calculated using a cross product and using a covariance matrix method.

The designated normal vectors (fig. 4), described in the three-dimensional space, have two angles defining their orientation relative to the reference plane $(x y)$. For each of these angles, you can then calculate the values of trigonometric functions such as sine, cosine or tangent, as well as their inverse, i.e. secans, cosecans and cotangent. By treating these two angular values as dependent, you can plot a threedimensional distribution (fig. 5) of angles or their trigonometric values for a given computational scale, and then - the values of statistics of such a distribution.

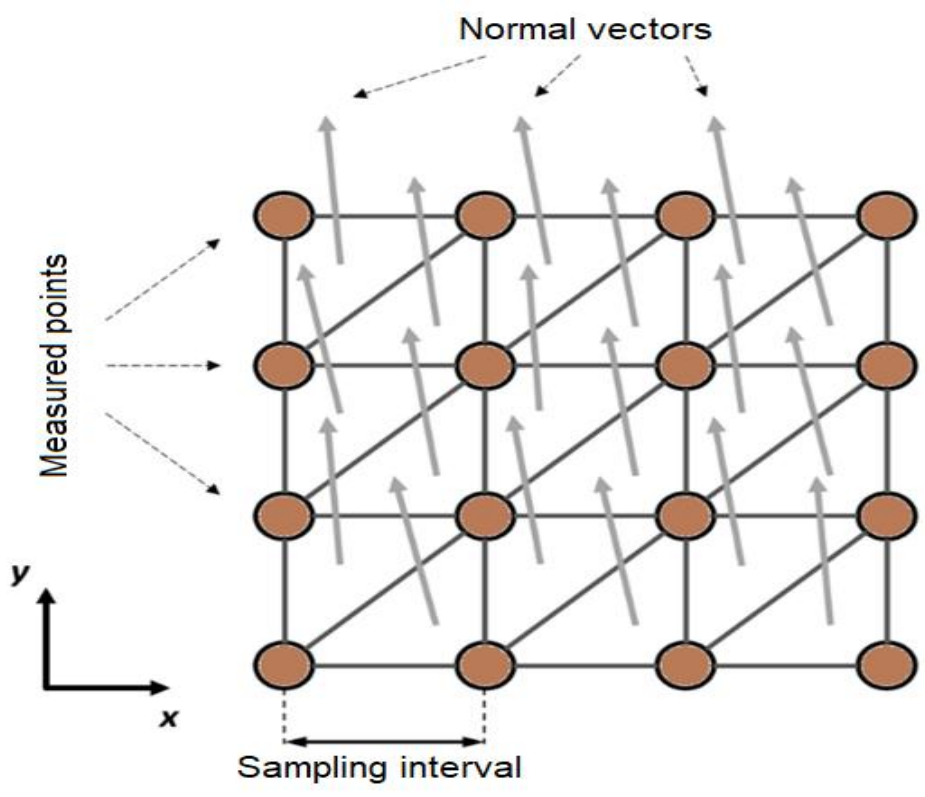

Fig. 3. Triangularization of measurement point clouds and calculated normal vectors for triangles

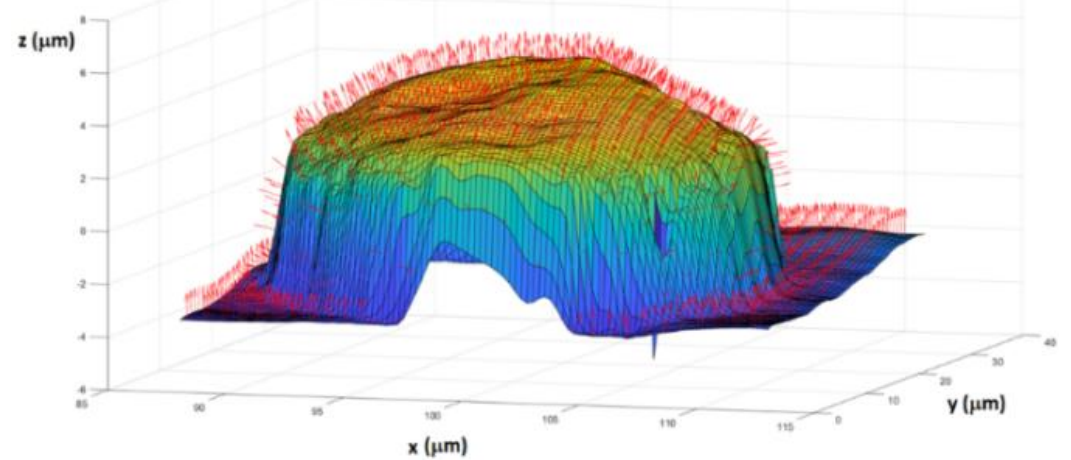

Fig. 4. Calculated normal vectors for a fragment of the surface of photographic paper (render thanks to the courtesy of David Etievant)
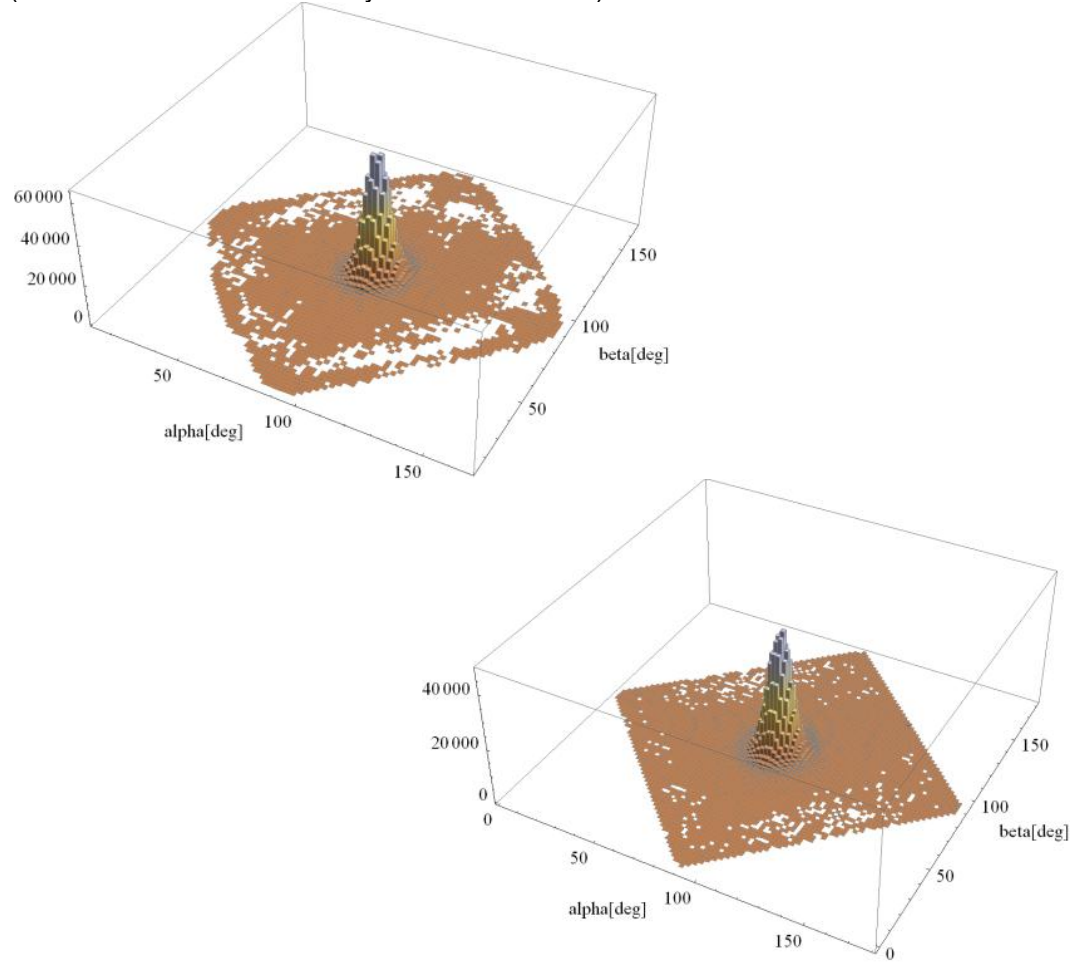

Fig. 5. Calculated distributions of multi-dimensional inclination angles of normal vectors for two different calculation scales 
Because these types of distributions belong to multidimensional group, it is recommended to use statistics for such issues, e.g. from: generalized variance, multivariate mean deviation, multivariate median deviation or multivariate kurtosis.

The method of multiscale slope analysis was used to analyze the topography of samples of various types of photographic paper. Strong correlations were observed $\left(R^{2}>0.9\right)$ between the parameter describing the gloss reflectance and the multivariate kurtosis for the angle of inclination of the normal vector, calculated using the cross product. Work on this issue is currently being implemented.

\section{Conclusions}

Currently, it is assumed that many more phenomena affect the surface topography or are directly dependent on it, than is proved by evidence in the form of analyzes, which undoubtedly confirm in a qualitative and quantitative manner the expected relations. Multiscale analysis is a tool that allows finding such dependencies in a functional way.

The development of geometric methods should contribute to a better understanding of the phenomena that govern the formation of topography as a result of physicochemical processes and surface interactions with the environment. On the other hand, a huge number of parameters describing surfaces are still insufficient to understand the complexity of this type of issues. Multiscale analysis requires - from researchers or users of commercial measurement systems - a broader knowledge of analysis or statistics. A step forward in popularizing these methods is the availability of such tools in the commercial software (e.g. MountainsMap), which has recently offered the possibility of conducting length-scale and area-scale analysis. The author hopes that the proposed multi-scale analysis will increase the interest of scientists and industry in this issue and will allow a better understanding of the nature of rough surfaces.

The research described in the article was financed by the Ministry of Science and Higher Education as part of staturory subsidy 02/22/DSPB/1431.

\section{REFERENCES}

1. Brown C.A., Hansen H.N., Jiang X.J, Blateyron F., Berglund J., Senin N., Bartkowiak T, Dixon B., Le Goïc G., Quinsat Y., Stemp W.J., Thompson M.K., Ungar P.S., Zahouani $\mathrm{H}$. "Multiscale analyses and characterizations of surface topographies". CIRP Annals Manufacturing Technology. 67, 2: pp. 839-862.

2. ISO 25178-2. Geometrical product specifications (GPS) - Surface texture: Areal - Part, 2.

3. Zahouani H., Lee S.-H., Vargiolu R. "The Multiscale Mathematical Microscopy of Surface Roughness, Incidence in Tribology, Lubrication at the Frontier: The Role of the Interface and Surface Layers in the Thin Film and Boundary Regime", pp. 379-390.

4. Jiang X., Blunt L., Stout K. "Three-dimensional surface characterization for orthopaedic joint prostheses". Proceedings of the Institution of Mechanical Engineers, Part $H$ : Journal of Engineering in Medicine. 213, 1, pp. 49-68.

5. Berglund J., Brown C.A., Rosén B-G., Bay N. "Milled die steel surface roughness correlation with steel sheet friction". Annals of the CIRP Manufacturing Technology. 59, 1, pp. 577-580.
6. Sayles R.S., Thomas T.R. "The spatial representation of surface roughness by means of the structure function: a practical alternative to correlation". Wear. 42, pp: 263-276.

7. Brown C.A., Charles P.D., Johnsen W.A., Chesters S. "Fractal analysis of topographic data by the patchwork method". Wear. 161, 1-2, pp. 61-67.

8. Underwood E.E., Banerji K. "Fractals in fractography". Materials Science and Engineering. 80, 1, pp. 1-14.

9. Bartkowiak T., Berglund J., Brown C.A. "Establishing functional correlations between multiscale areal curvatures and coefficients of friction for machined surfaces". Surface Topography: Metrology and Properties. 6(3), $9 \mathrm{~s}$.

10. Bartkowiak T., Lehner J.T., Hyde J., Wang Z., Pedersen D.B., Hansen H.N., Brown C.A. "Multi-scale areal curvature analysis of fused deposition surfaces". Proceedings ASPE 2015 Spring Topical Meeting: Achieving Precision Tolerances in Additive Manufacturing, pp. 77-82.

11. Bartkowiak T., Brown C.A. "A characterization of process-surface texture interac-tions in micro-electrical discharge machining using multiscale curvature tensor analysis". Journal of Manufacturing Science and Engineering, Transactions of the ASME. 140, 2, doi:10.1115/1.403760.

12. Zhang X., Drake N.A., Wainwright J., Mulligan M. "Comparison of slope estimates from low resolution DEMs: Scaling issues and a fractal method for their solution". Earth Surface Processes and Landforms. 24, 9, pp. 763-779.

13. Young G.C., Dey S., Rogers A.D., Exton D. "Cost and time-effective method for multiscale measures of rugosity, FD, and vector dispersion from coral reef 3D models". Plos One. 12, 4: e0175341.

Translation of scientific articles, their computer composition and publishing them on the website www.mechanik.media.p by original articles in Polish is a task financed from the funds of the Ministry of Science and Higher Education designated for dissemination of science.

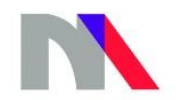

Ministry of Science and Higher Education

Republic of Poland 